\title{
Morphology, Kinematics and Star Formation in Spiral Galaxies in the Spitzer Survey of Stellar Structure in Galaxies $\left(\mathrm{S}^{4} \mathrm{G}\right)$
}

\author{
Santiago Erroz-Ferrer, ${ }^{1,2}$ Johan H. Knapen, ${ }^{1,2}$ Joan Font, ${ }^{1,2}$ \\ John E. Beckman ${ }^{1,2}$ and the $\mathbf{S}^{4} \mathbf{G}$ team \\ ${ }^{1}$ Instituto de Astrofísica de Canarias, Vía Láctea s/n 38205 La Laguna, Spain \\ ${ }^{2}$ Departamento de Astrofísica, Universidad de La Laguna, 38206 La Laguna, Spain \\ email: serroz@iac.es
}

\begin{abstract}
We present a study of the morphology, kinematics and star formation of a sample of 27 isolated spiral galaxies in the Spitzer Survey of Stellar Structure in Galaxies $\left(\mathrm{S}^{4} \mathrm{G}\right)$, on the basis of $\mathrm{H} \alpha$ Fabry-Perot (FP) data from the $\mathrm{GH} \alpha \mathrm{FaS}$ instrument at the William Herschel Telescope (WHT) in La Palma, complemented with images at 3.6 micron from the Spitzer Space Telescope, and with images in the $R$-band and in $\mathrm{H} \alpha$ taken with the ACAM instrument mounted on the WHT. With the FP data we can investigate the gas kinematics in terms of velocity maps and position-velocity diagrams, keys to constrain the secular evolution processes. We can also analyse the morphology and compute the star formation rate (SFR) with the ancillary data.
\end{abstract}

Keywords. galaxies: kinematics and dynamics - galaxies: spiral

\section{Motivation}

With this survey we can (a) perform a detailed study of the kinematic interplay of the ISM and regions of star formation, dust or other activity, (b) examine the secular evolution of the galaxy with kinematic studies, manifested in the structural components as spiral arms, bars, rings or lenses and (c) discuss possible deviations of the rotation curve that are caused by lopsidedness or asymmetries of the disk.

\section{Results}

In Erroz-Ferrer et al. (2012) (arXiv:1208.1409) we have presented the first results of this survey, a kinematical analysis of NGC 864. In the paper we have mainly analysed the kinematic data cubes. Also, we have used other ancillary data, like $R$-band and $\mathrm{H} \alpha$ images taken with the instrument ACAM in the WHT, IFU data with the SAURON instrument, also in the WHT, and we used the 3.6 micron $\mathrm{S}^{4} \mathrm{G}$ image.

The data cubes and velocity maps allow the study of the kinematics of every galaxy, including in-depth investigations of the rotation curve, velocity moment maps, velocity residual maps, gradient maps and position-velocity diagrams. In the residual maps, we have found that there are deviations from the circular rotation velocity, confirming the presence of non-circular motions along the bar. These are probably caused by the nonaxisymmetrical potential created by the bar. We can also observe the non-circular motions by creating a position-velocity diagram along the kinematic minor axis. In an ideal case without non-circular motions, the velocity profile along the kinematic minor axis would have been completely flat. This confirms that the bar has a significant influence on the kinematics of the galaxy, causing the velocities to deviate from the circular, rotational, motion. 